\title{
BMJ Open Speech and communication in Parkinson's disease: a cross-sectional exploratory study in the UK
}

\author{
Maxwell S Barnish, ${ }^{1}$ Simon M C Horton, ${ }^{1}$ Zoe R Butterfint, ${ }^{1}$ Allan B Clark, ${ }^{2}$ \\ Rachel A Atkinson, ${ }^{3}$ Katherine H O Deane ${ }^{1}$
}

To cite: Barnish MS, Horton SMC, Butterfint ZR, et al. Speech and communication in Parkinson's disease: a cross-sectional exploratory study in the UK. BMJ Open 2017;7:e014642. doi:10.1136/ bmjopen-2016-014642

- Prepublication history and additional material is available. To view please visit the journal (http://dx.doi.org/10.1136/ bmjopen-2016-014642).

Received 8 0ctober 2016 Revised 19 March 2017 Accepted 6 April 2017

\section{CrossMark}

${ }^{1}$ School of Health Sciences, Faculty of Medicine and Health Sciences, University of East Anglia, Norwich, UK ${ }^{2}$ Norwich Medical School, Faculty of Medicine and Health Sciences, University of East Anglia, Norwich, UK

${ }^{3}$ Adult Speech and Language Therapy Department, St Helier Hospital, Epsom and St Helier University Hospitals NHS Trust, London, UK

Correspondence to Dr Maxwell S Barnish; barnish@cantab.net

\section{ABSTRACT}

Objective To assess associations between cognitive status, intelligibility, acoustics and functional communication in PD.

Design Cross-sectional exploratory study of functional communication, including a within-participants experimental design for listener assessment.

Setting A major academic medical centre in the East of England, UK.

Participants Questionnaire data were assessed for 45 people with Parkinson's disease (PD), who had selfreported speech or communication difficulties and did not have clinical dementia. Acoustic and listener analyses were conducted on read and conversational speech for 20 people with $\mathrm{PD}$ and 20 familiar conversation partner controls without speech, language or cognitive difficulties. Main outcome measures Functional communication assessed by the Communicative Participation Item Bank (CPIB) and Communicative Effectiveness Survey (CES).

Results People with PD had lower intelligibility than controls for both the read (mean difference 13.7\%, $p=0.009$ ) and conversational (mean difference $16.2 \%$, $p=0.04$ ) sentences. Intensity and pause were statistically significant predictors of intelligibility in read sentences. Listeners were less accurate identifying the intended emotion in the speech of people with PD $(14.8 \%$ point difference across conditions, $p=0.02$ ) and this was associated with worse speaker cognitive status ( $16.7 \%$ point difference, $p=0.04$ ). Cognitive status was a significant predictor of functional communication using CPIB ( $\left.F=8.99, p=0.005, \eta^{2}=0.15\right)$ but not CES. Intelligibility in conversation sentences was a statistically significant predictor of CPIB $\left(F=4.96, p=0.04, \eta^{2}=0.19\right)$ and CES ( $\left.F=13.65, p=0.002, \eta^{2}=0.43\right)$. Read sentence intelligibility was not a significant predictor of either outcome.

Conclusions Cognitive status was an important predictor of functional communication - the role of intelligibility was modest and limited to conversational and not read speech. Our results highlight the importance of focusing on functional communication as well as physical speech impairment in speech and language therapy (SLT) for PD. Our results could inform future trials of SLT techniques for PD.

\section{INTRODUCTION}

Parkinson's disease (PD) affects around 1.5\% of people aged over 65 in Europe. ${ }^{1}$ Originally
Strengths and limitations of this study

- We provide the first same-study overview of associations at various stages along the potential pathway to reduced functional communication in Parkinson's disease (PD).

- Ours is the first study to consider the acoustic characteristics of the speech of British people with PD.

- Our study was cross-sectional and therefore cannot provide definitive insight into causality.

- Studies in this field, including ours, tend to have smaller sample sizes than many other fields in applied health science research, reflecting both the methodological challenges of speech analysis and the challenges of recruiting from this population.

conceptualised predominantly in terms of its motor features, ${ }^{2} \mathrm{PD}$ is now recognised to be a multifaceted condition. ${ }^{3}$ Indeed, non-motor symptoms, such as cognitive impairment affecting over a quarter of people with $\mathrm{PD},{ }^{4}$ are believed to exert a substantial effect on quality of life. ${ }^{5}$ Speech impairment, ${ }^{6}$ at the impairment level of the International Classification of Functioning (ICF), ${ }^{7}$ and functional communication difficulties, ${ }^{8}$ at the ICF activity and participation levels, are also widespread in PD. The mainstay of medical treatment for PD is levodopa-based pharmacotherapy, ${ }^{9}$ although non-adherence, ${ }^{10}$ dyskinesia ${ }^{11}$ and a lack of clear benefit on speech and cognition are problematic. ${ }^{12-14}$ Therefore, a wide range of supplementary therapies can be used, including singing, ${ }^{15}$ dance $^{16}$ and speech and language therapy (SLT). SLT is popular among people with PD and families alike ${ }^{17}$ but there is no definitive randomised controlled trial evidence for the effectiveness of currently tested SLT techniques. ${ }^{18}$ Moreover, the content and focus of SLT provision can vary markedly between localities. In the UK, the focus has traditionally been on motor function. In a survey conducted in 2007, functional communication was not reported to 
constitute a major part of many UK SLT's clinical practice for PD, ${ }^{19}$ although clinical contacts suggest that the situation has improved in recent years. Recently, MSB and SMCH published a clinical magazine feature article ${ }^{20}$ to emphasise the importance of functional communication to SLT clinicians.

Functional communication has been shown to be more important to people with PD than motoric speech impairment. ${ }^{21}$ Moreover, although it is an important predictor of quality of life, ${ }^{22}$ functional communication has received relatively limited research attention compared with motoric speech impairment. A systematic review of the literature up to July $2015^{23}$ found that nine studies prior to ours had assessed the association between cognitive status and functional communication in PD, of which eight had found a positive association. However, none had used a cognitive assessment sensitive to mild cognitive impairment in PD and a validated outcome measure that assessed either communicative effectiveness or communicative participation as a unified concept. Therefore, these studies may have failed to detect mild cognitive impairment short of dementia and also to accurately capture the concept of functional communication, resulting in potential inaccurate measurement of both independent and dependent variables. In addition, while three prior studies had found an association between intelligibility and communicative outcomes, only one study ${ }^{24}{ }^{25}$ used a standardised validated assessment tool-the Communicative Effectiveness Survey (CES). ${ }^{24}{ }^{25}$ However, CES covers the ICF activity level, not the ICF participation level. Subsequent to our review, one further large study ${ }^{22}$ has assessed functional communication outcomes in PD and found that people with PD with self-reported worse cognitive status and intelligibility had more difficulties in communicative participation. The size of this study is a major strength, but the study relied entirely on self-report data, which is a substantial limitation with regard to assessing cognitive status and intelligibility accurately.

Taking a wider perspective on communication difficulties in PD and potentially associated risk factors, it is important to note that no study in the published literature has provided an overview of the elements and potential mechanisms for change in the pathway from cognitive status and motoric speech impairment (acoustics) through reduced intelligibility to difficulties with emotional conveyance and functional communication in PD. There has been no comparative overview of which acoustic features are most predictive of reduced intelligibility. However, the available literature suggests that increased articulatory phonological distinctiveness ${ }^{26} 27$

Table 1 List of acoustic measures with a brief explanation of each

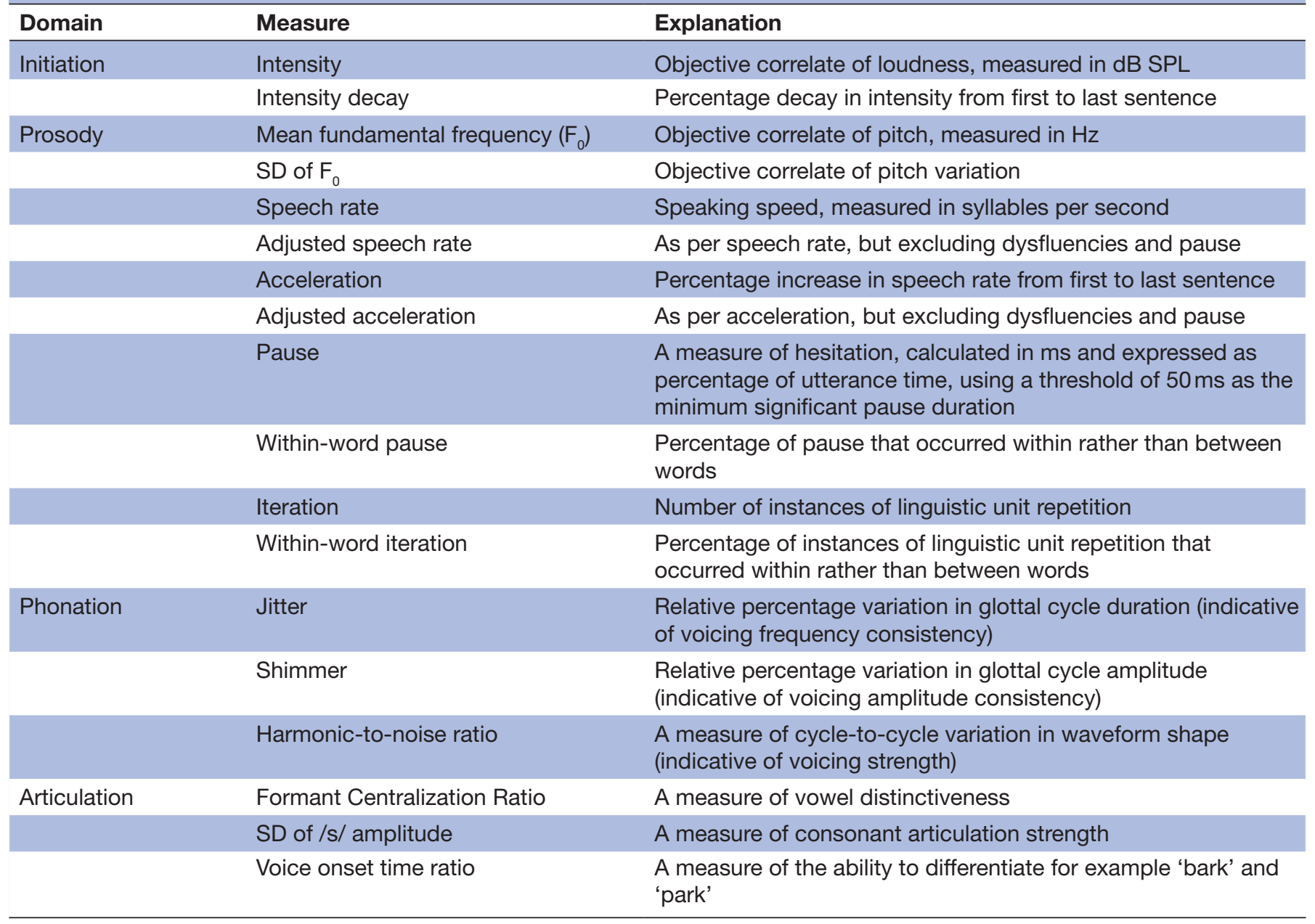


and loudness ${ }^{28} 29$ may be associated with better intelligibility, with the latter having beneficial effects on the distinctiveness of speech in PD besides loudness itself. ${ }^{29}$ Additionally, no study of speech acoustics has used speech that we considered to be naturalistic conversational dialogue-for example, the 'conversational' speech in the study by Goberman and Elmer ${ }^{30}$ was a standard passage read out in the style of conversational speech. Moreover, the ability to communicate emotions effectively is important in everyday life ${ }^{31}$ and studies have shown that reduced pitch variation and facial expression can cause negative evaluations of the personality of people with PD. ${ }^{32-35}$ Additionally, people with PD have been shown to have impaired perception of the intended emotion in the speech of others, ${ }^{36-38}$ which may relate at least partly to impaired mesolimbic processing. ${ }^{39}$ However, normal listeners' ability to identify specific emotions in the speech of people with PD has attracted limited research attention. Miller $e t \mathrm{al}^{40}$ showed that listeners were less likely to correctly identify the intended emotion in the speech of people with PD when auditory and visual information were both available. It was suggested that this effect may result from a lack of temporal synchronisation in the speech of people with PD. Meanwhile, Pell $e t a l^{41}$ also found reduced ability to identify emotions in the speech of people with PD, especially for anger and disgust, but did not assess presentation modality.

Informed by limitations in the existing literature, we conducted an exploratory study focusing on functional communication in PD as our primary outcome. This is an area that has received relatively little research attention, yet corresponds well to the priorities of people with PD. ${ }^{21}$ We conducted a study to provide an overview of associations along the potential pathway to functional communication difficulties in PD, since no prior study had done this. In addition, we added an aspect on emotional conveyance in order to further investigate the possibilities raised by Miller $e t a l^{40}$ especially with regard to presentation modality effects. Our key research questions for this study are as follows:

- How does cognitive status associate with functional communication in $\mathrm{PD}$, as measured by the Communicative Participation Item Bank (CPIB, primary research question) and CES?

Table 2 Key clinical characteristics of people with Parkinson's disease in the full and purposive samples

\begin{tabular}{lll}
\hline Measure & Full sample & Purposive sample \\
\hline $\begin{array}{l}\text { Disease duration } \\
\text { (years) }\end{array}$ & $6.5(8.3)^{\star}$ & $9.0(9.5)^{\star}$ \\
MoCA & $22.9(3.6)$ & $22.2(3.3)$ \\
HADS & $11.0(8.5)^{\star}$ & $9.6(4.8)$ \\
LEDD & $640.5(656.5)^{\star}$ & $691.5(1027.3)^{\star}$ \\
\hline
\end{tabular}

Figures are mean (SD), unless when marked with * in which case they are median (IQR).

HADS, Hospital Anxiety and Depression Scale; LEDD, Levodopa

Equivalent Daily Dose; MoCA, Montreal Cognitive Assessment.
What is the test-retest reliability and convergent validity of CPIB in our UK context?

- How does intelligibility, in both read and conversational sentences, associate with functional communication in PD?

- What were the acoustic differences between the speech of people with PD and conversation partners (CPs) in our sample; how did the intelligibility of these groups differ in read and conversational speech; and what acoustic factors predicted intelligibility outcomes?

- How did the emotional conveyance of people with PD and CPs differ, which mood contrasts were particularly affected, and did presentation modality (audio vs audiovisual) play a role?

\section{MATERIALS AND METHODS Design}

In order to assess associations along the potential pathway to functional communication difficulties in $\mathrm{PD}$, we used a cross-sectional design, into which we embedded a within-participants experimental design for listener assessment. Ours is a clinical linguistics/academic SLT study, rather than epidemiology. Our study also draws on some methods commonly used in psychology. Therefore, there is no suitable reporting guideline to follow. Ethical approval for this study was granted by the National Research Ethics Service Committee East of EnglandNorfolk. All requisite local governance approvals were obtained.

\section{Participants}

Our study recruited from the Neurology and Medicine for the elderly outpatient clinics at a major academic medical centre in the East of England region in 20122013. Patients were eligible for the study if they (1) were aged at least 18, (2) had idiopathic PD according to the UK Parkinson's Disease Society Brain Bank criteria, ${ }^{42}$ (3) had no clinical indication of dementia, (4) had no other serious medical conditions that would affect cognitive status or speech, (5) were not considered by clinical staff to be unsuitable for the study, for example, due to personal circumstances, (6) were native English speakers and (7) reported having some difficulty with their speech and/or communication. Participants with PD were asked to invite a familiar CP control to join them in the study where possible. CPs had to (1) be aged at least 18, (2) be a native English speaker, (3) not have PD and (4) not have any serious medical problems affecting cognition or speech. Written informed consent was obtained from all participants prior to the commencement of study procedures.

\section{Measures and data collection}

The study consisted of one appointment typically of around $45 \mathrm{~min}$ after consent, which could take place either at home or at the University of East Anglia. Initially, participants completed a demographic form, 
Table 3 Descriptive profile of principal speech and communication measures

\begin{tabular}{lll} 
& People with PD & $\begin{array}{l}\text { Conversation partner } \\
\text { controls }\end{array}$ \\
\hline Read sentence intelligibility & $81.1(15.0)$ & $87.9(3.6)$ \\
Conversational sentence intelligibility & $55.8(26.5)$ & $71.9(13.0)$ \\
\hline Emotional conveyance (happy audio) & $36.5(20.5)$ & $55.6(20.8)$ \\
Emotional conveyance (happy audiovisual, \%) & $54.1(20.5)$ & $61.4(13.9)$ \\
\hline Emotional conveyance (neutral audio, \%) & $55.4(18.0)$ & $46.7(18.6)$ \\
Emotional conveyance (neutral audiovisual,\%) & $38.5(25.3)$ & $53.6(20.8)$ \\
Emotional conveyance (sad audio, \%) & $55.8(21.3)$ & $64.8(18.7)$ \\
Emotional conveyance (sad audiovisual,\%) & $55.8(23.1)$ & $63.0(25.2)$ \\
CPIB (T score) & $53.0(9.6)$ & $\mathrm{NA}$ \\
CPIB (overall rating of degree to which PD affects & Not at all: $11(24 \%)$ & $\mathrm{NA}$ \\
communication, $\mathrm{n}(\%))$ & A little: $24(53 \%)$ & \\
& Quite a bit: $9(20 \%)$ & Very much: $1(2 \%)$ \\
\hline
\end{tabular}

Figures are mean (SD) unless stated. Intelligibility is scored as percentage of words correctly identified. Emotional conveyance is scored as percentage of tokens for which emotion was correctly identified.

CPIB, Communicative Participation Item Bank; PD, Parkinson's disease.

which for people with PD provided their medication information which allowed their Levodopa Equivalent Daily Dose $(\mathrm{LEDD})^{43}$ to be calculated. LEDD served as a proxy measure of non-speech-specific PD motor symptom severity. Validated assessments of cognitive status (Montreal Cognitive Assessment, MoCA),${ }^{4445} \operatorname{mood}$ (Hospital Anxiety and Depression Scale, HADS), ${ }^{46-48}$ communicative effectiveness (CES) 242549 and communicative participation (Communicative Participation Item Bank, CPIB $)^{50}$ were completed. CPIB was chosen as our primary measure of functional communication since it specifically assesses ICF participation level difficulties that have been shown to be most important to people with $\mathrm{PD},{ }^{21}$ and also has been thoroughly developed using item response theory methods ${ }^{50}$ and subsequent validated in PD in the USA and New Zealand, which are English-speaking countries. ${ }^{51}$ Therefore, we assessed testretest reliability by sending out a second copy of CPIB by post 2 weeks after the study visit and assessed convergent validity using CES in our UK setting. As per the terms of our ethical approval, cognitive, mood and functional communication assessments were only administered to participants with PD and not to CPs.

Audiovisual recordings were obtained of all participants' (PD and $\mathrm{CP}$ ) speech at a standardised distance of $1.5 \mathrm{~m}$ using Panasonic NV-GS17 (Panasonic, Osaka, Japan) video cameras. Video was encoded in high-quality $48 \mathrm{kHz}$ AVI format, from which high-quality $44.1 \mathrm{kHz}$ WAV audio files could be extracted. Participants first read a standardised set of 16 sentences taken from the Assessment of Intelligibility of Dysarthric Speech (AssIDS) assessment tool. ${ }^{52}$ Then, participants held a short conversation on a topic of their choice in an exercise that was intended to offer as naturalistic speech as possible. Besides offering support to people with PD in completing questionnaires where required, this was the main advantage of including familiar CPs in the study-King and Gallegos-Santellan have shown that people with dysarthria use different strategies with familiar and unfamiliar conversation partners. ${ }^{53}$ Finally, participants read four standardised sentences in three ways: happy, sad and neutral. All sentences contained words of moderate-to-high frequency and did not have an intrinsic emotional connotation. Three of the sentences were taken with permission from Miller $e t a t^{40}$ namely 'The cake is too yellow', 'You dropped the sausages in the trifle' and 'Sam is not a dog'. One further sentence was generated by the research team: 'He went to the park'.

\section{Data analysis}

Speech sample analysis (acoustics, intelligibility and emotional conveyance) was conducted on a purposive sample of 20 people with PD and 20 CPs. In order to generate our purposive sample, first, any samples that suffered from technical failure, other issues such as road noise and non-compliance with the task instructions were excluded. Then, selection sought to achieve a balanced profile of demographic and clinical features among people with PD and maximise comparability of demographics between the $\mathrm{PD}$ and $\mathrm{CP}$ groups, within the bounds of what was available in our sample. Only people with PD who provided a CP were considered. Age, gender, accent and perceived severity of speech disorder were also considered in selection. In particular, it was important to ensure generalisability of the PD sample. As we used standardised read sentences in the intelligibility assessment, we designed this part of the study so that each script sentence would only be rated twice by each assessor in order to avoid stimulus exposure effects and learning bias. ${ }^{54}$ Assessment of self-report measures could be conducted on the full sample of 45 people with PD, but could not be conducted on CPs as we did not gather these data for ethical reasons. 
Acoustic (phonetic) analysis was conducted by MSB using Praat software (P. Boersma \& D. Weenink, University of Amsterdam) and a reliability check of a randomly selected $10 \%$ sample of acoustic data points drawn from 10 different participants $(25 \%$ of the phonetic analysis sample size) was completed by Senior Lecturer in Phonetics, ZRB. Acoustic measures covered four broad domains ${ }^{56-58}$-initiation (the production of airflow), prosody (rhythm and melody), phonation (voicing) and articulation (the modification of sound waves by the resonant properties arising from different vocal tract configurations). A list of measures with a brief description of each is provided in table 1. Sentence-level parameters were calculated for conversational and mood sentences. Phoneme-level parameters were additionally calculated for the set of 16 standardised read sentences.

Sixty-four assessors ( $88 \%$ female, median age 22) served as members of the study team to conduct assessment of speech samples for intelligibility and emotional conveyance. Assessors had to be (1) members of the University of East Anglia (UEA, for ethical reasons), (2) fluent English speakers and (3) not having significant expertise in listening to disordered speech (eg, SLT staff, final-year SLT students and those with a close member with PD or working with groups or individuals with PD as part of their course or extracurricular activities). Twenty tracks (each comprising a different combination of utterances and speakers) were created in EditStudio software (MediaChance, Ottawa, Canada) with stimulus allocation based on a Latin Square design ${ }^{59}$ and randomised presentation order. All tracks were rated three times and four tracks were rated an additional time, meaning that each token spoken by each participant was rated by at least three different assessors. The intelligibility task was transcription and following AssIDS protocol, the outcome measure was percentage of words correctly identified. This was scored separately for read and conversational sentences and the transcript for the latter was agreed between authors MSB and SMCH. The emotional conveyance task was to circle which of three options (happy, neutral or sad) the speaker intended to convey and the outcome measure was percentage of moods correctly identified following Miller et $a l .{ }^{40}$ In the intelligibility task, all stimuli were presented audiovisually, while in the emotional conveyance tasks, half were presented audiovisually and half in audio only. In all listener assessment tasks, assessors could only listen to each sentence once and sentences from people with PD and CPs were matched for length. The rationale for including an audio-only condition in the emotional conveyance assessment was to test the preliminary finding by Miller $e t a l^{40}$ that listeners were less likely to correctly identify the intended emotion in the speech of people with PD when auditory and visual information were both available. In contrast, for intelligibility assessment, we wanted to replicate the most common real-life listening conditions through presenting audiovisual information.

Statistical aspects of the study were overseen by Senior Lecturer in Medical Statistics, ABC. The sample size was powered on the primary research question, to assess the relationship between cognitive status and CPIB scores in people with PD. Since this is an association rather than a group difference, we used a power calculation tool for observational designs. ${ }^{60}$ The effect size to use for the power calculation was determined by senior statistician $\mathrm{ABC}$ informed by (1) preliminary systematic literature searches by the research team that later became our systematic review ${ }^{23}$ and (2) the research team's combined wider theoretical, scientific and clinical knowledge and expertise about communication in neurological conditions such as PD, which both informed us to expect a moderate relationship between cognitive status and functional communication in PD. Therefore, a power calculation was run to state the minimum number of people with PD we would require to have $80 \%$ power to detect an expected association equivalent to $\mathrm{r}=0.5$ for this relationship. This gave us an uncorrected target sample size of 30 . Following discussion with our steering committee, as approved by the ethics board, we decided to recruit a minimum of 40 and a maximum of 45 people with PD as our attrition and exclusion corrected target sample size. The reasons for seeking to recruit a minimum of 10 additional participants (33\%) above the minimum target from the power calculation were that (1) we were recruiting from an older, often frail population whose clinical severity we did not know ahead of the study visit for ethical reasons; (2) our study population was a group that may experience high fatigue, making the risk of non-completion of the study visit difficult to predict; (3) the potential of withdrawal of scheduled participants due to death, serious illness or other personal reasons; (4) the potential that scheduled participants may be ineligible or unwilling/unable to give informed consent at the study visit; and (5) the fact that our study involved making audiovisual recordings in participants' homes where our speech science experts predicted an elevated risk of technical failure and issues with recording quality, for example, due to pets or road noise. Stata (Stata, College Station, Texas, USA) and SPSS (IBM, Armonk, New York, USA) software were used for statistical analysis. Appropriate linear regression models were constructed to assess (1) differences in speech acoustics between people with PD and CPs and the contribution of cognitive status to speech acoustics of people with PD; (2) differences in intelligibility and the contribution of cognitive status and particular acoustic characteristics; (3) differences in the acoustic correlates of happy, neutral and sad mood and the contribution of cognitive status; (4) differences in emotional conveyance and the contribution of cognitive status and particular acoustic characteristics; (5) the contribution of cognitive status and intelligibility to functional communication as measured by CES and CPIB. The test-retest reliability of CPIB was assessed using interclass correlation and its convergent validity with CES using correlation. Due to the exploratory nature of the study, the fact that analysis was on a range of outcome measures rather than repeated analysis of the same outcome measure and the fact that all 
comparisons were planned in advance, it was decided a priori not to perform adjustment for multiple testing. ${ }^{61} \mathrm{~A}$ $p$ value of $p<0.05$ was considered significant and variables associated at $\mathrm{p}<0.1$ were retained in models as marginally significant. There were limited missing data, only one participant had missing data for the CPIB outcome measure and none for CES. Full-case analysis was used.

\section{RESULTS}

\section{Participants}

Forty-five people with PD and 29 CPs were recruited. Forty-five people with PD contributed to the questionnaire analysis. The mean age was 71.0 (SD 8.1), 28 (62\%) were male and the most common educational category was to have no formal educational qualifications $(n=17$, $38 \%)$.

Among the 20 people with PD whose data were used for speech sample analysis, the mean age was 71.1 (SD 9.0), $23(65 \%)$ were male and the most common educational category was shared between no formal educational qualifications and vocational qualifications (both $\mathrm{n}=7,35 \%$ ). Table 2 presents the clinical characteristics of both the full $(n=45)$ and purposive $(n=20)$ samples of people with PD.

Among the $20 \mathrm{CPs}$ whose data were used for speech sample analysis, the mean age was 70.0 (SD 10.4), 7 (35\%) were male and the most common educational category was to have vocational qualifications $(n=8,40 \%)$.

\section{Speech acoustics and intelligibility}

Table 3 profiles the principal speech and communication measures in our study. The overall concordance rate was $\mathrm{r}=0.99$ for inter-rater reliability of acoustic measures. In read sentences, people with PD had lower speech intensity and greater pause time than CPs. For other measures, there was either no significant difference, a marginally significant difference or an effect that applied only for one gender. MoCA was associated with intensity, although the effect was in opposite directions for men and womenmen with PD with better cognitive status spoke more loudly, while women with PD with better cognitive status spoke more quietly. MoCA was not associated with pause. In conversational sentences, people with PD had higher within-word iteration than CPs. This was not associated with MoCA. Statistical details on the main effects and interactions can be found in online supplementary tables 1 (read sentences) and 2 (conversational sentences).

Assessors were significantly less accurate in transcribing both the read (mean difference $=13.7$ percentage points, $\mathrm{p}=0.009$ ) and conversational (mean difference $=16.2$ percentage points, $\mathrm{p}=0.04$ ) speech of people with PD compared with CPs. In neither case was there an association between MoCA and intelligibility. In read sentences, intensity (mean difference $=2.4$ percentage points per $\mathrm{dB}$ SPL, $\mathrm{p}=0.04$ ) and pause (mean difference $=3.6$ percentage points per percentage unit change in pause, $\mathrm{p}=0.04$ ) were identified as significant predictors of listener accuracy-assessors were more accurate in transcribing the read speech of people with PD who spoke more loudly and paused less. No significant acoustic predictors of conversational sentence intelligibility were identified. Gender was not a statistically significant predictor of intelligibility.

\section{Emotional conveyance}

In the emotion sentences, men with PD spoke more quietly than CPs, women with PD had significantly reduced mean fundamental frequency compared with CPs, both men and women with PD had significantly reduced SD of fundamental frequency, men with PD had significantly reduced speech rate (but not adjusted speech rate) and both men and women with PD had significantly increased pause time. In the PD group, participants with MoCA below median had significantly lower speech rate and adjusted speech rate. Main effects of mood were found within the PD group for most measures, meaning that people with PD were on the whole able to distinguish emotions in their speech, although distinctions were reduced relative to CPs. Significant and marginally significant group by emotion interactions, for happy versus sad, suggests that people with PD were particularly impaired in the production of happy emotion. Statistical details on the main effects and interactions can be found in online supplementary table S3.

Listeners were significantly less accurate in identifying the intended emotion (happy, neutral or sad) in the speech of people with PD compared with CPs (mean difference $=14.8$ percentage points, $\mathrm{p}=0.04$ ). A significant interaction between group and emotion (mean difference for group * emotion (sad vs happy) $=17.8$ percentage points, $\mathrm{p}<0.001$ ) shows that the impact of PD on listener accuracy was greater for happy mood. There was no significant effect of presentation modality (audiovisual vs audio only) on listener accuracy. There was a significant effect of MoCA (mean difference $=16.7$ percentage points between participants scoring above and below the median, $\mathrm{p}=0.04$ ), showing that listeners had more difficulty in identifying emotion in the speech of people with $\mathrm{PD}$ with greater cognitive impairment. A significant interaction between MoCA and emotion (mean difference for MoCA (median split) $*$ emotion (sad vs happy) $=23.2$ percentage points, $\mathrm{p}=0.009$ ), showing that the differential effect of PD on happy mood conveyance was less for those with more intact cognition.

CPIB showed satisfactory test-retest reliability $(r=0.85$, $\mathrm{p}<0.001)$ and validity $(\mathrm{r}=0.74, \mathrm{p}<0.001)$ in our population, noting that CPIB and CES are measures of related but not identical constructs, so a higher concordance would have been unexpected. In the full sample, MoCA $\left(\mathrm{F}=8.99, \mathrm{p}=0.005, \eta^{2}=0.15\right)$ and HADS $(\mathrm{F}=8.73$, $\left.\mathrm{p}=0.005, \eta^{2}=0.15\right)$ were retained as significant predictors of CPIB, while HADS ( $\left.\mathrm{F}=20.18, \mathrm{p}<0.001, \eta^{2}=0.32\right)$ was the only significant predictor of CES, but there was also a marginally significant finding for LEDD ( $\mathrm{F}=3.72$, $\left.\mathrm{p}=0.06, \eta^{2}=0.06\right)$. With regard to MoCA subdomains, the 
executive and visuospatial $\left(\mathrm{F}=3.22, \mathrm{p}=0.08, \eta^{2}=0.05\right)$ and attention $\left(\mathrm{F}=3.05, \mathrm{p}=0.09, \eta^{2}=0.05\right)$ subdomains were both marginally significant predictors of CPIB. Among the purposive sample for whom intelligibility scores were available, MoCA $\left(\mathrm{F}=5.32, \mathrm{p}=0.04, \eta^{2}=0.20\right)$ and intelligibility in conversational sentences $\left(\mathrm{F}=4.96, \mathrm{p}=0.04, \eta^{2}\right.$ $=0.19)$, but not intelligibility in read sentences, were significant predictors of CPIB, while only intelligibility in conversational sentences $\left(\mathrm{F}=13.65, \mathrm{p}=0.002, \eta^{2}=0.43\right.$ ) was a significant predictor of CES.

\section{DISCUSSION}

The study presented in this article is the first to provide an overview of associations along the potential pathway from cognitive status and motoric speech impairment (acoustics) through reduced intelligibility to difficulties with emotional conveyance and functional communication in PD. We also include a combination of self-reported and observed measures, an approach which avoids one of the key limitations associated with larger studies, such as that by McAuliffe $e t a l^{22}$ that only include self-report measures. Ours is also the first to study the acoustics of the speech of British people with PD, mindful that there are notable acoustic differences between British and American English. ${ }^{62} 63$

The first main finding was that intelligibility was reduced in both read and conversational speech for people with PD compared with controls, and the effect was greater on conversational sentences, potentially reflecting the greater cognitive and perceptual challenges of spontaneous speech. The second main finding was that acoustic differences between people with PD and CPs in our sample were modest and few were statistically significant, although many participants in our study had relatively mild motoric speech difficulties. The results of our study reflect the natural hierarchy that can emerge in clinical practice, starting initially with work on physical aspects of read speech due to the cognitive demands of altering one's speech and then progressing to less structured tasks that generalise more readily to everyday conversation (RA Atkinson, personal communication, 2016).

The third main finding was that emotional conveyance, especially of happy emotion, was impaired in people with PD compared with CPs. The fourth main finding was that, despite a relatively mild profile of motoric speech deficits, participants often had difficulties with functional communication. Intelligibility did not account for a large proportion of variance in functional outcomes, emphasising the need to account for and include other elements in functional communication tasks in SLT for people with PD to overcome the challenge with generalisation from the clinic to everyday life. Cognitive status predicted CPIB and emotional conveyance, but not intelligibility or CES. This may imply a greater role for cognitive status with regard to participation-level phenomena.

Our identification of reduced intelligibility in people with PD compared with CPs is in line with previous studies and in particular our identification of intensity as a key predictor of intelligibility (although only found for read sentences in our study) corroborates the prior findings of Tjaden and Sussman ${ }^{28}$ and Neel, ${ }^{29}$ while our identification of pause suggests a potentially novel acoustic correlate of intelligibility in PD. Our study is the first to compare conversational and read speech intelligibility in PD and found that intelligibility was lower in conversational sentences, which is explicable in terms of contextual effects and the lower distinctiveness of more spontaneous speech and therefore the potential for a lower ability on the behalf of listeners to adjust for phonetic alterations.

With regard to emotional conveyance, in keeping with Miller et $a l^{40}$ and Pell et $a l^{41}$ our findings support the view that people with PD were less successful in conveying emotion in their speech. Our findings show that the communication of happy emotion was particularly affected, although our study cannot confirm the mechanisms which might be causing this effect. Unlike Miller $e t a l,{ }^{40}$ potentially due to lesser severity of speech impairment, we did not find that listeners were more accurate in the audio only condition compared with the audiovisual condition. Our identification that intelligibility contributes a relatively modest proportion of the variance in functional communication is consistent with Donovan $e t a l,{ }^{2425}$ although we advance this knowledge by demonstrating differences between conversational and read sentence intelligibility as well as communicative effectiveness and communicative participation. Previous studies in our review ${ }^{23}$ and also McAuliffe $e t a l^{22}$ have generally found an association between cognitive status and functional communication. The prior study by Miller $e t a l,{ }^{664}$ which did not find such as association, used as a measure of cognitive status the Mini Mental State Examination, ${ }^{65}$ which has been shown to be insensitive to mild cognitive impairment in PD. ${ }^{66-69}$

Communication is fundamental to humanity and in particular the development and maintenance of human relationships. ${ }^{70}$ Although participation may mean different things to different people ${ }^{71}$ it is evident that participation aspects, including those of functional communication, ${ }^{21}$ are of great importance to people with PD. Indeed, it is important that research and clinical priorities and perspectives match those of people with the condition as closely as possible. ${ }^{72}$ The relatively modest contribution of intelligibility to functional communication outcomes shown by our study and others indicates that it is important for SLT for people with PD to focus on non-motoric issues affecting functional communication in addition to more traditionally recognised motoric issues. In environments where there has been a move to include a higher proportion of functional communication in therapy, this should be maintained. In environments where this has not yet happened, it is recommended that greater focus on functional communication be considered. In achieving this, it is important to consider what the particular client's communication needs and goals are, what challenges the client faces in accomplishing these 
and what approaches may facilitate this. It is important to remember that communication needs differ between clients, and that clients differ in what they consider full participation in life. ${ }^{71}$ Further research is required to investigate the effectiveness of SLT for PD. The pathway proposed by our study could be useful to inform future research into defining treatments to include in intervention trials. In addition, it is important to conduct further research into the gender-specific aspects of communication difficulties in PD, which have received limited research attention.

There are some limitations of this study that should be taken into account. The PhD time scale did not allow us to undertake a longitudinal study, so we cannot be definitive about causality. Second, it was not possible to use the entire sample size for speech sample analysis due to the constraints that read sentences impose on the sample size in the intelligibility assessment so as to avoid learning biases. Third, the sample we recruited had on average relatively mild motoric speech deficits, potentially due to greater reluctance to take part in speech studies among those with more severe speech impairment or alternatively due to an over-representation of people with early PD and greater insight into research. Fourth, reflecting both the methodological challenges of speech analysis and the challenges of recruiting from this population, sample sizes in this field tend to be lower than in many other areas of applied health research. Fifth, we were unable to measure motor disability directly. However, we offered LEDD as a proxy measure of motor disability to models assessing functional outcomes in order to minimise confounding by motor disability. Moreover, some studies have shown that cognitive impairment can be common in people with PD who are early on the motor decline pathway. ${ }^{73}{ }^{74}$ However, LEDD has limitations as a proxy measure of motor status. For example, one study ${ }^{75}$ found no significant association between LEDD and Hoehn and Yahr staging. Therefore, future studies should consider assessing how scores from explicit motor assessments, such as the Universal Parkinson's Disease Rating Scale, ${ }^{, 76}$ predict functional communication outcomes. Sixth, due to regulatory constraints, we were unable to conduct further analyses following the Chief Investigator's departure from the host institution on completion of his PhD. Without this restriction, we may have been able to consider whether different modelling or reporting options may have been preferable.

In conclusion, we present the first study that provides an overview of the potential pathway from cognitive status and motoric speech impairment through reduced intelligibility to difficulties with emotional conveyance difficulties and functional communication in PD. Our results support the idea that SLT for people with PD should focus on functional communication as well as motor deficits, and could also inform future trials to identify the optimal form of therapy. The pathway to functional communication difficulties in PD is likely to involve complex, multifactorial mechanisms for change, including motoric, cognitive and psychosocial elements. Since our study is exploratory, future confirmatory research is required to validate and extend our findings. This should include clarification of the elements and mechanisms of this pathway, as well as how they may differ between individuals with PD, which is a condition known to vary considerably in its clinical expression. ${ }^{77}$

Acknowledgements This study constituted MSB's PhD work at the University of East Anglia (UEA). The authors thank Dr Carolyn Baylor, Professor Kathryn Yorkston and colleagues (University of Washington, USA) for prior information about the CPIB tool. The authors thank our participants and assessors, our recruiting clinicians at the Neurology and Medicine for the Elderly Clinics at the Norfolk and Norwich University Hospital as well as Mike Stevens, formerly of UEA, who provided guidance and training in relation to audiovisual technology used in the study.

Contributors MSB was the Chief Investigator and KHOD the primary academic supervisor. SMCH and RAA are registered speech and language therapies and are specialists in adult neurological disorders. The study was conceptualised and overseen by MSB, SMCH, ZRB and KHOD. Data were collected by MSB. Acoustic analysis was conducted by MSB and ZRB. Listener assessment was conducted by our panel of assessors supervised by MSB with advice from SMCH, ZRB and KHOD. Statistical analysis was overseen by $A B C$ and conducted by MSB and ABC. The first draft of the manuscript was written by MSB. Data were initially interpreted by MSB and further interpretation provided by SMCH, ZRB, ABC, RAA and KHOD. All authors contributed academically and/or clinically valuable revisions to the manuscript. All authors approved the submission.

Funding We acknowledge funding from a UEA PhD studentship to MSB.

Competing interests None declared.

Patient consent No patient identifiable information in the manuscript.

Ethics approval Ethical approval for this study was granted by the National Research Ethics Service Committee East of England - Norfolk. All requisite local governance approvals were obtained.

Provenance and peer review Not commissioned; externally peer reviewed.

Data sharing statement We confirm that no additional data are available.

Open Access This is an Open Access article distributed in accordance with the Creative Commons Attribution Non Commercial (CC BY-NC 4.0) license, which permits others to distribute, remix, adapt, build upon this work non-commercially, and license their derivative works on different terms, provided the original work is properly cited and the use is non-commercial. See: http://creativecommons.org/ licenses/by-nc/4.0/

(C) Article author(s) (or their employer(s) unless otherwise stated in the text of the article) 2017. All rights reserved. No commercial use is permitted unless otherwise expressly granted.

\section{REFERENCES}

1. von Campenhausen S, Bornschein B, Wick R, et al. Prevalence and incidence of Parkinson's disease in Europe. Eur Neuropsychopharmacol 2005;15:473-90.

2. Parkinson J. An essay on the shaking palsy. J Neuropsychiatry Clin Neurosci 2002;14:223-36.

3. Gopalakrishna A, Alexander SA. Understanding Parkinson disease: a complex and multifaceted illness. J Neurosci Nurs 2015;47:320-6.

4. Litvan I, Aarsland D, Adler $\mathrm{CH}$, et al. MDS Task Force on mild cognitive impairment in Parkinson's disease: critical review of PDMCl. Mov Disord 2011;26:1814-24.

5. Duncan GW, Khoo TK, Yarnall AJ, et al. Health-related quality of life in early Parkinson's disease: the impact of nonmotor symptoms. Mov Disord 2014;29:195-202.

6. Ho AK, lansek R, Marigliani C, et al. Speech impairment in a large sample of patients with Parkinson's disease. Behav Neurol 1999;11:131-7.

7. World Health Organization. International classification of functioning, disability and Health. Geneva: World Health Organization, 2001.

8. Miller N, Noble E, Jones D, et al. How do I sound to me? Perceived changes in communication in Parkinson's disease. Clin Rehabil 2008;22:14-22.

9. Worth PF. How to treat Parkinson's disease in 2013. Clin Med 2013;13:93-6. 
10. Daley DJ, Myint PK, Gray RJ, et al. Systematic review on factors associated with medication non-adherence in Parkinson's disease. Parkinsonism Relat Disord 2012;18:1053-61.

11. Nutt JG. Clinical pharmacology of levodopa-induced dyskinesia. Ann Neurol 2000;47(4 Suppl 1):S160-4.

12. Plowman-Prine EK, Okun MS, Sapienza CM, et al. Perceptual characteristics of Parkinsonian speech: a comparison of the pharmacological effects of levodopa across speech and non-speech motor systems. NeuroRehabilitation 2009;24:131-44.

13. Skodda S, Visser W, Schlegel U. Short- and long-term dopaminergic effects on dysarthria in early Parkinson's disease. J Neural Transm 2010;117:197-205.

14. Seppi K, Weintraub D, Coelho M, et al. The movement disorder society evidence-based medicine review update: Treatments for the non-motor symptoms of Parkinson's disease. Mov Disord 2011;26:S42-S80.

15. Barnish J, Atkinson RA, Barran SM, et al. Potential Benefit of singing for people with Parkinson's Disease: A systematic review. $J$ Parkinsons Dis 2016;6:473-84.

16. Sharp K, Hewitt J. Dance as an intervention for people with Parkinson's disease: a systematic review and meta-analysis. Neurosci Biobehav Rev 2014:47:445-56.

17. Miller N, Noble E, Jones D, et al. Survey of speech and language therapy provision for people with Parkinson's disease in the United Kingdom: patients' and carers' perspectives. Int J Lang Commun Disord 2011;46:179-88

18. Herd CP, Tomlinson CL, Deane KHO, et al. Speech and language therapy versus placebo or no intervention for speech problems in Parkinson's disease. Cochrane Database Syst Rev 2012;8:CD002812.

19. Miller N, Deane KH, Jones D, et al. National survey of speech and language therapy provision for people with Parkinson's disease in the United Kingdom: therapists' practices. Int J Lang Commun Disord 2011;46:189-201.

20. Barnish M, Horton S. The factors affecting the speech of people with Parkinson's Disease study team. Cognitive impairment and communication. Bulletin: the official magazine of the Royal College of Speech and Language Therapists, 2016; July 11.

21. Miller N, Noble E, Jones D, et al. Life with communication changes in Parkinson's disease. Age Ageing 2006;35:235-9.

22. McAuliffe MJ, Baylor CR, Yorkston KM. Variables associated with communicative participation in Parkinson's disease and its relationship to measures of health-related quality-of-life. Int $J$ Speech Lang Pathol 2016 [Epub ahead pof print: 27 June 2017] doi:10.1080/17549507.2016.1193900.

23. Barnish MS, Whibley D, Horton SM, et al. Roles of cognitive status and intelligibility in everyday communication in people with Parkinson's Disease: A systematic review. J Parkinsons Dis 2016:6:453-62.

24. Donovan NJ, Kendall DL, Young ME, et al. The communicative effectiveness survey: preliminary evidence of construct validity. Am J Speech Lang Pathol 2008;17:335-47.

25. Donovan NJ. Extending dysarthria research with a measure of communicative effectiveness, $\mathrm{PhD}$ Dissertation, University of Florida. 2005. http://etd.fcla.edu/UF/UFE0010055/donovan_n.pdf (accessed 7 Oct 2016).

26. Weismer G, Jeng JY, Laures JS, et al. Acoustic and intelligibility characteristics of sentence production in neurogenic speech disorders. Folia Phoniatr Logop 2001:53:1-18

27. Tjaden K, Wilding GE. Rate and loudness manipulations in dysarthria: acoustic and perceptual findings. J Speech Lang Hear Res 2004:47:766-83.

28. Tjaden K, Sussman J. Perception of coarticulatory information in normal speech and dysarthria. J Speech Lang Hear Res 2006;49:888-902.

29. Neel AT. Effects of loud and amplified speech on sentence and word intelligibility in Parkinson disease. J Speech Lang Hear Res 2009:52:1021-33.

30. Goberman AM, Elmer LW. Acoustic analysis of clear versus conversational speech in individuals with Parkinson disease. $J$ Commun Disord 2005;38:215-30.

31. Planalp S. Communicating emotion: social, moral, and cultural processes. Cambridge: Cambridge University Press, 1999.

32. Tickle-Degnen L, Lyons KD. Practitioners' impressions of patients with Parkinson's disease: the social ecology of the expressive mask. Soc Sci Med 2004:58:603-14

33. Pentland B, Gray JM, Riddle WJ, et al. The effects of reduced nonverbal communication in Parkinson's disease. Br J Disord Commun 1988;23:31-4

34. Pentland B, Pitcairn TK, Gray JM, et al. The effects of reduced expression in Parkinson's disease on impression formation by health professionals. Clin Rehabil 1987;1:307-12.
35. Jaywant $A$, Pell MD. Listener impressions of speakers with Parkinson's disease. J Int Neuropsychol Soc 2010;16:49-57.

36. Möbes J, Joppich G, Stiebritz F, et al. Emotional speech in Parkinson's disease. Mov Disord 2008;23:824-9.

37. Benke T, Bösch S, Andree B. A study of emotional processing in Parkinson's disease. Brain Cogn 1998;38:36-52.

38. Schröder C, Möbes J, Schütze M, et al. Perception of emotional speech in Parkinson's disease. Mov Disord 2006;21:1774-8.

39. Schott BH, Niehaus L, Wittmann BC, et al. Ageing and earlystage Parkinson's disease affect separable neural mechanisms of mesolimbic reward processing. Brain 2007;130:2412-24.

40. Miller N, Jones D, Lewis A. Perception of speech in Parkinson's disease: seeing and hearing the difference [Conference presentation]. Parkinson's UK Research Conference, York, UK, 2008.

41. Pell MD, Cheang HS, Leonard CL. The impact of Parkinson's disease on vocal-prosodic communication from the perspective of listeners. Brain Lang 2006;97:123-34.

42. Gibb WR, Lees AJ. The relevance of the Lewy body to the pathogenesis of Idiopathic Parkinson's disease. J Neurol Neurosurg Psychiatry 1988;51:745-52.

43. Tomlinson CL, Stowe R, Patel S, et al. Systematic review of levodopa dose equivalency reporting in Parkinson's disease. Mov Disord 2010;25:2649-53.

44. Nasreddine ZS, Phillips NA, Bédirian V, et al. The Montreal Cognitive Assessment, MoCA: a brief screening tool for mild cognitive impairment. J Am Geriatr Soc 2005;53:695-9.

45. Chou KL, Amick MM, Brandt J, et al. A recommended scale for cognitive screening in clinical trials of Parkinson's disease. Mov Disord 2010;25:2501-7.

46. Zigmond AS, Snaith RP. The hospital anxiety and depression scale Acta Psychiatr Scand 1983;67:361-70.

47. Schrag A, Barone P, Brown RG, et al. Depression rating scales in Parkinson's disease: critique and recommendations. Mov Disord 2007;22:1077-92.

48. Leentjens AF, Dujardin $\mathrm{K}$, Marsh L, et al. Anxiety rating scales in Parkinson's disease: critique and recommendations. Mov Disord 2008;23:2015-25.

49. Donovan NJ, Velozo CA, Rosenbek NJ. The communicative effectiveness survey: investigating its item-level psychometric properties. J Med Speech Lang Pathol 2007;15:433-47.

50. Baylor C, Yorkston K, Eadie T, et al. The communicative participation Item Bank (CPIB): item bank calibration and development of a disorder-generic short form. J Speech Lang Hear Res 2013;56:1190-208.

51. Baylor C, McAuliffe MJ, Hughes LE, et al. A differential item functioning (DIF) analysis of the communicative participation Item Bank (CPIB): comparing individuals with Parkinson's disease from the United States and New Zealand. J Speech Lang Hear Res 2014:57:90-5.

52. Yorkston KM, Beutelman DR. Assessment of Intelligibility of Dysarthric Speech. Austin: Pro-Ed, 1981.

53. King JM, Gallegos-Santellan MS. Strategy use by speakers with dysarthria and both familiar and unfamiliar conversation partners. $J$ Med Speech Lang Pathol 1999;7:113-21.

54. Bornstein RF, D'Agostino PR. Stimulus recognition and the mere exposure effect. J Pers Soc Psychol 1992;63:545-52.

55. Grill-Spector K, Henson R, Martin A. Repetition and the brain: neural models of stimulus-specific effects. Trends Cogn Sci 2006;10:14-23.

56. Fant G. The source filter concept in voice production. Speech Music and Hearing Quarterly Status and Progress Report, KTH Royal Institute of Technology, Stockholm, Sweden 1981;22:21-37.

57. Catford JC. A practical introduction to phonetics. Oxford University Press: Oxford, 2001.

58. Huckvale M. Data processing: digital analysis of speech audio signals, in Müller N, Ball MJ (Eds), Research methods in clinical linguistics and phonetics. Chichester: Wiley Blackwell, 2013.

59. Grant DA. The Latin Square principle in the design and analysis of psychological experiments. Psychol Bull 1948;45:427-42.

60. Arsham H. Sample size determination. http://home.ubalt.edu/ ntsbarsh/business-stat/otherapplets/samplesize.htm. (accessed 7 Oct 2016).

61. Bender R, Lange S. Adjusting for multiple testing--when and how? J Clin Epidemiol 2001;54:343-9.

62. Yan Q, Vaseghi S. A comparative analysis of UK and US English accents in recognition and synthesis. Proc IEEE Int Conf Acoustic Speech Signal Process. . Orlando, 2002:1. 413-6.

63. Yan Q, Vaseghi S. Analysis, modeling and synthesis of formants of British, American and Australian accents. Proc IEEE Int Conf Acoust Speech Signal process. . Hong Kong, 2003:1. 712-5. 
64. Miller N, Andrew S, Noble E, et al. Changing perceptions of self as a communicator in Parkinson's disease: a longitudinal follow-up study. Disabil Rehabil 2011;33:204-10.

65. Folstein MF, Folstein SE, McHugh PR. "Mini-mental state". A practical method for grading the cognitive state of patients for the clinician. J Psychiatr Res 1975;12:189-98.

66. Hoops S, Nazem S, Siderowf AD, et al. Validity of the MoCA and MMSE in the detection of $\mathrm{MCl}$ and dementia in Parkinson disease. Neurology 2009;73:1738-45.

67. Mamikonyan E, Moberg PJ, Siderowf A, et al. Mild cognitive impairment is common in Parkinson's disease patients with normal Mini-Mental State Examination (MMSE) scores. Parkinsonism Relat Disord 2009;15:226-31.

68. Zadikoff C, Fox SH, Tang-Wai DF, et al. A comparison of the mini mental state exam to the Montreal cognitive assessment in identifying cognitive deficits in Parkinson's disease. Mov Disord 2008;23:297-9.

69. Dalrymple-Alford JC, MacAskill MR, Nakas CT, et al. The MoCA: well-suited screen for cognitive impairment in Parkinson disease. Neurology 2010;75:1717-25.
70. Douglas M, Ney S. Missing persons: a critique of personhood in the social sciences. Berkeley and London: University of California Press, 1998.

71. Hammel J, Magasi S, Heinemann A, et al. What does participation mean? an insider perspective from people with disabilities. Disabil Rehabil 2008;30:1445-60.

72. Petit-Zeman S, Firkins L, Scadding JW. The James Lind Alliance: tackling research mismatches. Lancet 2010;376:667-9.

73. Yarnall AJ, Breen DP, Duncan GW, et al. Characterizing mild cognitive impairment in incident Parkinson disease: the ICICLE-PD study. Neurology 2014;82:308-16.

74. Pedersen KF, Larsen JP, Tysnes OB, et al. Prognosis of mild cognitive impairment in early Parkinson disease: the Norwegian ParkWest study. JAMA Neurol 2013;70:580-6.

75. Yoritaka A, Shimo Y, Takanashi M, et al. Motor and non-motor symptoms of 1453 patients with Parkinson's disease: prevalence and risks. Parkinsonism Relat Disord 2013;19:725-31.

76. Movement Disorder Society Task Force on Rating Scales for Parkinson's Disease. The Unified Parkinson's Disease Rating Scale (UPDRS): status and recommendations. Mov Disord 2003;18:738-50.

77. Wolters ECh. Variability in the clinical expression of Parkinson's disease. J Neurol Sci 2008;266:197-203. 